models of SUI, for the regeneration of the rhabdosphincter and urethral submocosa.

In total, 119 patients with SUl and no more than mild hypermobility of the urethra and bladder completed 1 year of follow-up. Autologous myoblasts and fibroblasts were grown from biopsy samples of biceps muscle, and transurethral ultrasonography was used to guide their injection into the rhabdosphincter (myoblast suspension) and the urethral submucosa (fibroblast-collagen suspension). No complications were associated with the implantation procedure and no severe adverse effects were reported during the follow-up period.

At 1 year, 94 (79\%) of the 119 patients were completely continent. The remaining patients showed substantial (13\%) or slight $(8 \%)$ improvement of continence; no deterioration of incontinence was observed. Compared with pretreatment measurements, improvements were seen at 1 year in Incontinence Score and Incontinence Quality of Life score, and also in the thickness, contractibility and electromyographic activity of the rhabdosphincter, representing clinical improvement in sphincter function. The improvements could not be attributed to a simple 'bulking effect' of the injections.

The investigators conclude that transurethral-ultrasonography-guided injection of autologous myoblasts and fibroblasts is a safe and effective treatment for female SUI, and that long-term multicenter replication of their data is warranted.

Original article Mitterberger M et al. (2007) Autologous myoblasts and fibroblasts for female stress incontinence: a 1-year follow-up in 123 patients. BJU Int 100: 1081-1085

\section{Transobturator method of treating SUI preserves normal clitoral blood flow}

Tension-free midurethral sling procedures are now the preferred treatment for stress urinary incontinence (SUI) in women, as they are highly effective yet minimally invasive; however, risks associated with tension-free vaginal tape (TVT) include injury to the bladder, bowel and blood vessels. The transobturator (TOT) approach decreases the risk of complications, but whether it helps to preserve sexual function is unknown.

In a prospective, open clinical study, Caruso et al. monitored vascular function in the area around the urethra and clitoris in women with
SUI before and after TVT or TOT. Translabial color Doppler ultrasonography was performed in 42 women undergoing TVT surgery, and in 63 women undergoing TOT. Blood flow in the clitoral arteries was measured before the procedure and 6 months later.

After treatment, women in the TVT group had a significantly lower mean pulsatility index and mean peak systolic velocity, and a significantly higher mean resistance index than before treatment, indicating a reduced and less-efficient blood flow after surgery. In the TOT group, however, values were similar before and after the procedure. TOT procedures were shorter than TVT because cystoscopy was not required, and no complications were reported (versus two bladder perforations in the TVT group). Objective cure rates were high in both the TOT and TVT groups (95.0\% and $88.2 \%$, respectively).

The authors conclude that the TOT procedure might be preferable to TVT in women with SUI because, as well as providing good cure rates for stress urinary incontinence, it conserves clitoral blood flow after treatment.

Original article Caruso S et al. (2007) Clitoral blood flow changes after surgery for stress urinary incontinence: pilot study on TVT versus TOT procedures. Urology 70: 554-557

\section{High survival rate after Mohs micrographic surgery for penile cancer}

Mohs micrographic surgery (MMS) for dermatological cancers involves sequential tissue excision under microscopic control, with review of sections by the surgeon to ensure negative margins. The last case series reported of MMS for penile cancer was published in 1987; therefore, to provide a contemporary report, Shindel et al. reviewed their experience of penile cancer treatment with the technique.

The researchers identified 33 patients treated with MMS (41 procedures in total) for penile cancer at Washington University School of Medicine from 1988-2006. Five procedures were terminated because of urethral involvement or lesion size. The mean number of surgical stages was $2.6 \pm 1.4$, and the mean postoperative defect size was $3.6 \times 3.0 \mathrm{~cm}$. Primary repair or granulation was used to correct 13 defects, 4 were repaired by skin grafts and 25 were repaired with tissue flaps and urethroplasty. Follow-up data for 25 patients were available; 